\title{
Hardware Testbed for Sidelink Transmission of 5G Waveforms without Synchronization
}

\author{
David Garcia-Roger, Josue Flores de Valgas, Jose F. Monserrat, N. Cardona \\ Universitat Politècnica de València, iTEAM Research Institute \\ Valencia, Spain \\ Email: \{dagarro,joflode1,jomondel,ncardona\}@iteam.upv.es
}

\author{
Nicolo Incardona \\ Politecnico di Milano \\ Milano, Italy \\ Email: nicolo.incardona@mail.polimi.it
}

\begin{abstract}
This paper details a hardware testbed conceived for studying the impact of the lack of synchronism between transmitters on several 5G waveform candidates, which is of special relevance for car-to-car communications. The experimental results show that a proper fitting of waveforms in a software-defined platform would permit increasing the range of communication between cars without peer-to-peer synchronization, paving the way for the real development of collision avoidance messages.
\end{abstract}

\section{INTRODUCTION}

IEEE 802.11p, also known as wireless access in the vehicular environment (WAVE), is an amendment to the IEEE 802.11 standard that extends its applicability to vehicular environments, including short-range communications for data exchange between vehicles (V2V) and between vehicles and the roadside infrastructure. IEEE $802.11 \mathrm{p}$ is based on orthogonal frequency-division multiplexing (OFDM) [1], being its multiple access counterpart, OFDMA, widely used to divide the spectrum in multiple and orthogonal parallel sub-bands, as it happens in long term evolution (LTE) technology [2]. However, as it turns out, the orthogonality property of OFDMA is only verified under ideal conditions, that is to say, under perfect frequency synchronization and precise time alignment during the cyclic prefix $(\mathrm{CP})$. This issue imposes critical requirements, in such a way that, in order not to introduce inter-carrier interference (ICI) or inter-symbol interference (ISI), the terminals should be prevented from transmitting if a central entity does not verify that both time and frequency alignment are being respected. However, in the current design of LTE-Vehicle (LTE-V) the synchronization requirements are becoming a crucial issue. For example, while vehicles may be synchronized with elements of the roadside infrastructure (i.e. base stations), it is not expected that the synchronism between vehicles in a V2V sidelink communication scenario would be supported by such elements; thus specific synchronization signals are now under discussion for the support of this direct V2V communication. Moreover, the concept of timing advance (TA) may not be reused because it only assists in the determination of the distance to a common element (the base station) and is useless when determining the distance between two vehicles with varying relative distances. Consequently, it is of the utmost benefit to assess alternatives to OFDM, which should be able to tolerate a lower degree of synchronization without introducing additional ICI or ISI.
The filter bank multi-carrier (FBMC) [3] and the universal filtered OFDM (UF-OFDM), also known as universal filtered multi-carrier (UFMC) [4], modulation schemes are two of the alternative fifth-generation $(5 \mathrm{G})$ waveform candidates currently under consideration for designing a multicarrier physical (PHY) layer. Like OFDM, FBMC and UF-OFDM schemes segment the spectrum into multiple orthogonal sub-bands. However, unlike OFDM, FBMC performs a per-subcarrier filtering and UF-OFDM applies a filter to blocks of subcarriers. As a result, with FBMC and UF-OFDM the sidelobes are attenuated and thus the ICI and ISI issues are less critical than with OFDM for transmissions not perfectly synchronized.

5G waveform candidates have been subject of research efforts for several years, but so far mainly from the theoretical viewpoint, with only a few experimental testbeds [5]-[7], which do not address the synchronization requirements of the studied waveforms. This paper is focused on using a hardware testbed to evaluate a set of $5 \mathrm{G}$ waveform candidates, explaining the usefulness of the proposed hardware testbed in checking the feasibility of each candidate for sidelink communications, and collecting the results of evaluating the impact of the lack of synchronization and MIMO performance.

The remaining sections of the paper are structured as follows. Section II describes the waveforms implemented, briefly introducing OFDM, FBMC, and UF-OFDM. Section III explains the V2V scenario studied in the testbed. Section IV gives an overview of the experimental hardware testbed. Section $\mathrm{V}$ provides additional details about its software components and Section VI presents the measurement results. Finally, Section VII draws the main conclusions of this work.

\section{WAVEFORMS UNDER STUDY}

\section{A. OFDM}

A transmitter uses a baseband constellation mapper to map a bit stream to a QAM symbol called $X . N$ parallel QAM symbols $X[0], X[1], \ldots, X[N-1]$ represent the symbols transmitted at each subcarrier. By modulating these symbols with the inverse fast Fourier transform (IFFT) the transmitter generates the discrete baseband OFDM symbol

$$
x[n]=\mathcal{F}^{-1}\{X[i]\}=\frac{1}{\sqrt{N}} \sum_{i=0}^{N-1} X[i] e^{j \frac{2 \pi}{N} n i}, 0 \leq n \leq N-1,
$$


where $N$ is the number of subcarriers, and $X[i]$ is the symbol on the $i$-th subcarrier. The receiver recovers the symbol $\hat{X}[i]$ by using the fast Fourier transform (FFT) as follows

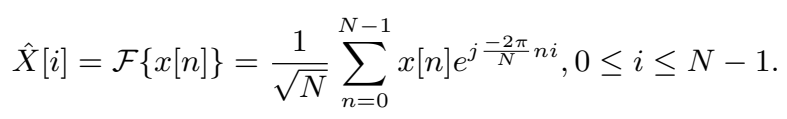

\section{B. $O Q A M / F B M C$}

Multicarrier with offset QAM (OQAM) FBMC is chosen because of its maximum spectral efficiency [3]. It allows subcarrier bands that are maximally overlapped and minimally spaced (carrier spacing equal to the symbol rate).

The transmitter maps a bit stream to an OQAM symbol on the $i$-th carrier of the $m$-th frame denoted by $X_{m}[i]$. OQAM splits the symbols into their real and imaginary parts, modulates the in-phase and quadrature components by half a symbol period, and runs the FFT at twice the rate, with real and imaginary symbols alternating both in time and frequency axis. In contrast to OFDM, it is not orthogonal with respect to the complex plane, and each subcarrier introduces interference to its neighboring subcarriers. OQAM allows the easy cancellation of this interference at the receiver by ignoring the part of the received symbol not carrying data.

FBMC filters on a per subcarrier basis by means of a prototype filter, which is characterized by the overlapping factor $K$, the number of multicarrier symbols which overlap in the time domain. $K$ is also the amount of frequency coefficients which are introduced between the FFT filter coefficients. Prototype filters are designed to transmit data in such a manner that no ISI occurs. In the testbed, the prototype filter in [8], [9] generates a filter bank via frequency shifts. Note that subcarriers with odd or even indexes do not overlap; only the neighbor subcarriers have influence in a certain subcarrier.

At the transmitter, the symbols $X_{m}[i]$ are converted to the time domain by means of an IFFT, obtaining the output $x_{m}[n]$,

$$
x_{m}[n]=\mathcal{F}^{-1}\left\{X_{m}[i]\right\}=\frac{1}{\sqrt{N}} \sum_{i=0}^{N-1} X_{m}[i] e^{j 2 \pi n i / N} .
$$

The prototype filter is applied to these symbols before transmitting. To that effect, $x_{m}[n]$ is duplicated $K$ times and then multiplied with the impulse response of the prototype filter. Each filtered frame symbol $y_{m}[n]$ is

$$
y_{m}[n]=p[n] x_{m}[n \bmod N],
$$

where $p[n]$ is the impulse response of length $L_{p}=N K$ of the prototype filter (given by applying the IFFT to the frequency response of the prototype filter).

All the filtered frame symbols must be shifted by half a symbol period $N / 2$, obtaining the final transmitted signal $y[n]$,

$$
y[n]=\sum_{m=1}^{K} y_{m}\left[n-\frac{m-1}{2} N\right] .
$$

The shift extends the total length of $y_{n}$ to $L_{p}+N(K-$ 1) $/ 2=N(3 K-1) / 2$ samples. Typical lengths of four times the symbol period are used because the frequency response of the filter needs to be rather tight. In turn, long ramp up and ramp down areas are required in case of bursty data transmissions. Both the fact that subcarriers are interferring into their neighborhood, and the need for long filters cause various issues, especially if practical aspects of programming such waveform on a hardware testbed are considered.

The received signal $r[n]$ is divided into $K$ frames and the prototype filter is applied to each frame. Note that the $m$ th frame verifies that $r_{m}[n]=r\left[n+\frac{m-1}{2} N\right]$. Therefore, by multiplying the $m$-th frame with the impulse response of the prototype filter, the following is obtained:

$$
s_{m}[n]=p[n] r_{m}[n] .
$$

The $K$ symbols reassemble again into one symbol, $\hat{s}_{m}[n]$,

$$
\hat{s}_{m}[n]=\sum_{k=0}^{K-1} s_{m}[n+k N], n=0,1, \ldots, N-1 .
$$

note that at the transmitter the symbol after the IFFT was originally duplicated $K$ times. At this point, an FFT operation is applied to the symbol $\hat{s}_{m}[n]$, frame by frame, rebuilding the OQAM symbol $\hat{X}_{m}[i]$,

$$
\hat{X}_{m}[i]=\mathcal{F}\left\{\hat{s}_{m}[n]\right\}=\frac{1}{\sqrt{N}} \sum_{n=0}^{N-1} \hat{s}_{m}[n] e^{-j 2 \pi n i / N} .
$$

\section{UF-OFDM}

UF-OFDM is a generalization of FBMC and OFDM. While FBMC filters at the subcarrier level and OFDM filters the entire band, UF-OFDM filters blocks of subcarriers (called sub-bands).The entire bandwidth is thus divided into $B$ subbands and each sub-band is allocated with $n_{k}$ consecutive subcarriers, i.e. a sub-band is like the physical resource block (PRB) concept of LTE. With an $N$-point IFFT operation, and filling only the corresponding subcarriers, the frequency domain signal of the $k$-th sub-band, $X_{k}$, is transformed into the time domain as $x_{k}$. Then, this output signal is filtered by a finite impulse response (FIR) filter $f_{k}$ of length $L$ adapted to the $k$-th sub-band. The filtering process results in a signal of length of $N+L-1$ because of the linear convolution between $x_{k}$ and $f_{k}$. Then, all the filtered sub-band signals $y_{k}$ are added together and transmitted, resulting in $\sum_{k=1}^{B} y_{k}$.

At the receiver, FFT-based detection transforms the received signal to the frequency domain. Since the UF-OFDM symbol has length $N+L-1$, zero-padding is needed to perform the $2 N$-point FFT. The estimated symbol $\hat{X}[i]$ is derived from this $2 N$-point FFT by taking only the even subcarriers, since odd subcarriers contain interference from all data symbols.

The classical cyclic prefix OFDM (CP-OFDM) is a special case of UF-OFDM with $L=1$. Within a single sub-band, spectral properties for UF-OFDM are like filtered OFDM, while different sub-bands are spectrally separated as FBMC separates single subcarriers. UF-OFDM does not require a cyclic prefix but may use it to further improve ISI protection. The filter length depends on the sub-band width. Integer multiples of 12 for the number of subcarriers combined into a single sub-band are typical, which corresponds with the 


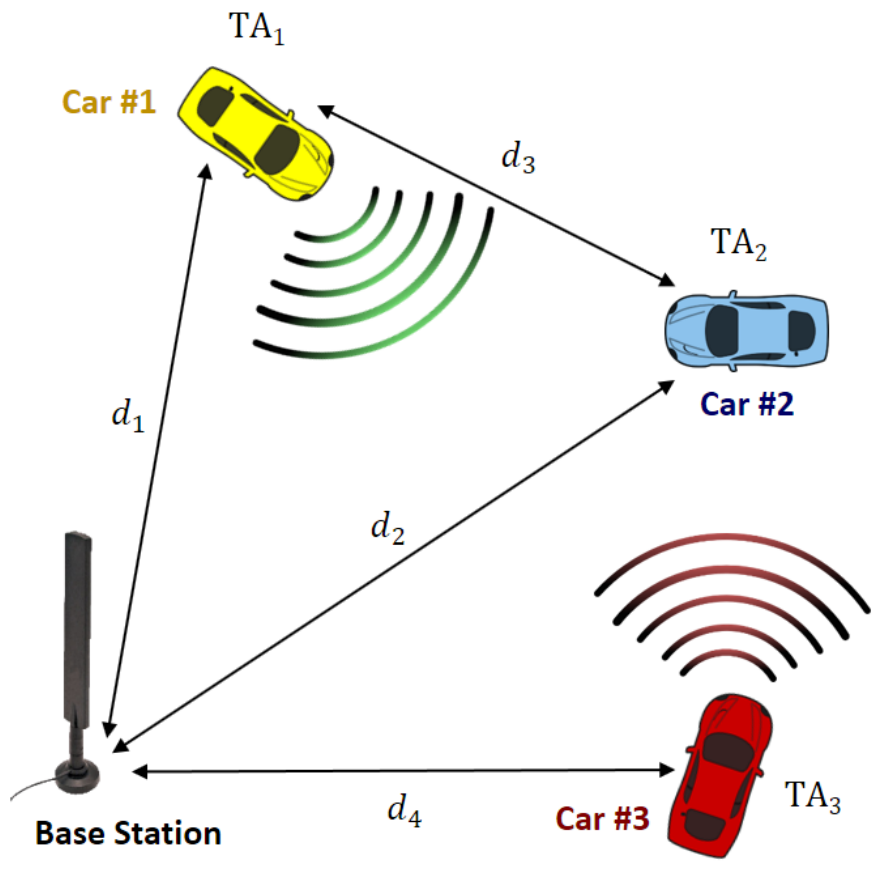

Fig. 1. V2V case scenario.

LTE numerology for one or several PRBs. The advantage of larger sub-bands is that the filter tail becomes shorter, reducing interference between UFMC symbols. Moreover, requiring UF-OFDM filter lengths in the range of the CP is also typical, resulting in about $7 \%$ overhead.

\section{V2V SCENARIO}

This paper studies the effect of timing offset (TO) in OFDM, OQAM/FBMC, and UF-OFDM systems with respect to the sidelink three-user scenario depicted in Fig. 1. As shown, the three vehicles are synchronized with respect to the base station via an ongoing closed-loop TA control enforced for each user in the uplink, thus anticipating the transmission so as to achieve perfect synchronization at the base station. TA is calculated as TA $=2 d / c$, being $d$ the distance in meters between the base station and the vehicle and $c$ the speed of light. If opportunistic sidelink communications are allowed, vehicles are free to transmit data between them at will. Under such conditions, time-aligning each user before the transmission can start is unacceptable, and thus supporting multiple concurrent $\mathrm{V} 2 \mathrm{~V}$ transmissions renders impossible for vehicles to be perfectly synchronized between them. In fact, asynchronous operation may be necessary to reach an acceptable level of spectral efficiency.

This work assumes that vehicles lack a dedicated synchronization mechanism between them, only synchronizing with the base station and using the same TA for their sidelink communications. In Fig. 1, if car \#1 and car \#2 do not modify their TA, then the TO experienced at car \#2 is $\mathrm{TO}=$ $\mathrm{TA}_{1}-\mathrm{TA}_{2}+\left(d_{3} / c\right)$. Sidelink communication opportunities distributed in time and frequency mapped into consecutive

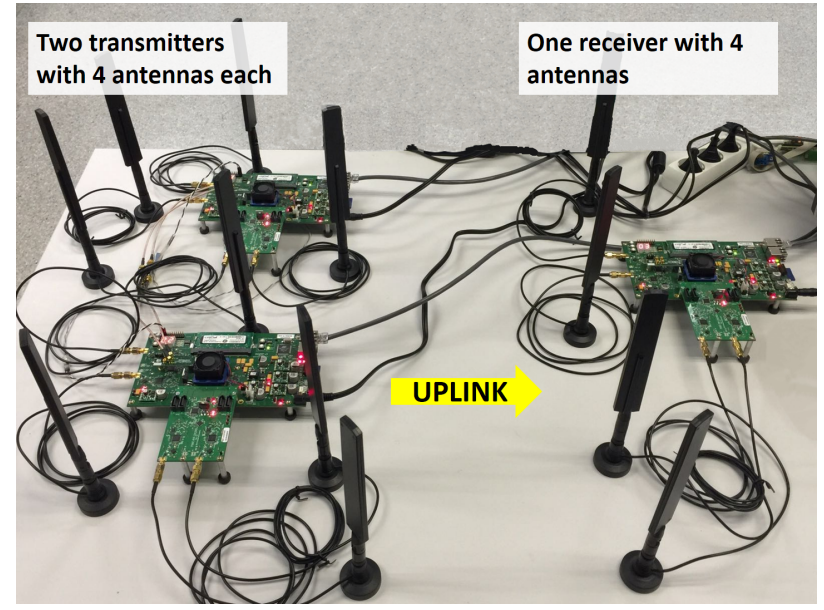

Fig. 2. 5G Waveforms Hardware Testbed.

symbols and adjacent frequency resources produce TO that will be perceived by the receiver as multiuser interference.

Previous work has evaluated the impact of timing synchronization for OFDM systems (analytically [10], or jointly with simulations [11]); for OQAM/FBMC (either mathematically [12], or combining analytical evaluation and numerical simulations [13], [14]; and for UF-OFDM (analysis and simulation [15]). Moreover, previous comparisons between waveforms have been done, again via numerical analysis and simulation: examining OFDM and FBMC [16], and studying OFDM, FBMC and UF-OFDM (among others) [17]. In contrast, the experiments in the present paper are tested and validated through a real channel under real transmission conditions, in a wireless communication system using readily available RF communication hardware boards implementing the IEEE $802.11 \mathrm{p}$ protocol. The findings in the present paper are in agreement with previous analytical and numerical contributions, complementing the state of the art by assessing the impact of the TO from the hardware platform viewpoint.

\section{HARDWARE TESTBED}

The hardware testbed is shown in Fig. 2. It consists of three WARP v3 kit boards by Mango Communications [18]. Two of them are configured as transmitters and the other one as receiver. Each board supports up to four antennas each.

The WARP v3 kit board includes a Xilinx Virtex-6 LX240T field programmable gate array (FPGA) for the real-time transmission and reception of waveforms. Each board has access to four MAX2829-based radio interfaces with a $2.4 / 5 \mathrm{GHz}$ transceiver of up to $40 \mathrm{MHz}$ of $\mathrm{RF}$ bandwidth, and up to $20 \mathrm{dBm}$ of transmission power. Each radio interface implements a full digital-to-RF transceiver chain; the analog to digital conversion is performed by an Analog Devices AD9963, and between baseband and RF by a Maxim MAX2829.

For the communication with the boards, a computer with the 64-bit version of Windows 8.1 Enterprise installed on a two core $2.4 \mathrm{GHz}$ processor with $4 \mathrm{~GB}$ of RAM is running MATLAB 2015b. The computer has a dedicated Gigabit 
Ethernet PCI-E NIC. The three WARP v3 kits are connected to each other and to the computer through the 1 Gigabit-Ethernet LAN ports of a TP-LINK TL-WR1043ND router.

\section{SOFTWARE-DEFInEd RAdio Programming}

The software developed runs with the WARP hardware testbed in the loop. It makes use of the WARPLab framework for rapid PHY prototyping and coordination of multipleantenna communication boards. WARPLab uses MATLAB to control the boards and perform signal pre/post-processing, while moving time critical components with strict latency requirements into the FPGA. It generates an IEEE 802.11p frame consisting of three different fields: PLCP preamble, signal and multiple data, as shown in Fig. 3. The PLCP preamble consists of a short (STS) and a long training sequence (LTS). The STS is a repetition of 10 equal short symbols of length 16 samples. The LTS comprises 2 identical sequences, of length 64 samples each, preceded by a 32-sample guard interval (GI). The synchronization process is split into a coarse TO and carrier frequency offset (CFO) estimation based on the LTS, followed by a fine estimation. The coarse TO estimation is accomplished with the algorithm in [19], currently a standard algorithm in OFDM systems. This delay-and-correlate algorithm exploits the periodicity of the training symbols. The coarse CFO estimation is performed with the correlation algorithm in [19]. After this coarse estimation, subsequent samples are multiplied by the CFO error estimates. After that, the program obtains an exact starting point at the first sample of the first LTS, which would be critical for the decoding process involving the FFT operation, moreover this starting point is useful because the LTS is also needed for channel estimation. This is achieved with cross-correlation or matched filter method, which correlates a local stored STS symbol with an already frequency-synchronized received signal. The output of the LTS correlator is a peak arising at the end of each of the two LTS symbols. The correlator implemented is a SignSign [20]. Fine carrier frequency offset estimation is performed with the aid of four pilot carriers. Finally, the data field contains multiple payload symbols carried by the waveforms analyzed in this paper. Note that the hardware testbed performs synchronization since the asynchronous situations are forced in a controlled manner by adding a certain shift into the signal samples. This is achieved via a clock module which enables sourcing and sinking the sampling and RF reference clocks directly to the WARP v3 clock buffers, hence the cable connection synchronizes the clocks at the two transmitters and allows shared triggering of the FPGA designs.

\section{Vi. Performance Results}

The performance comparison of the waveforms studied involved a total of 1000 measurements per waveform using the PHY layer parameters of IEEE 802.11p shown in Table I. In each case, the values of CP (OFDM), the overlap factor $K$ (OQAM/FBMC), and sidelobe attenuation (UF-OFDM) are explicitly stated in the figures. The modulation used is 16QAM. Note that we refer in the legends to dynamic OFDM
TABLE I

KEY 802.11P PHY LAYER PARAMETERS USED

\begin{tabular}{|l||c|}
\hline Parameters & IEEE 802.11p \\
\hline \hline FFT size & 64 \\
Total subcarriers & 52 \\
Data subcarriers & 48 \\
Pilot subcarriers & 4 \\
Symbol duration & $8 \mu \mathrm{s}$ \\
Guard interval & $1.6 \mu \mathrm{s}$ \\
FFT period & $6.4 \mu \mathrm{s}$ \\
Preamble Duration & $32 \mu \mathrm{s}$ \\
Subcarrier frequency spacing & $0.15625 \mathrm{MHz}$ \\
Channel bandwidth & $10 \mathrm{MHz}$ \\
Band & $5.9 \mathrm{GHz}$ \\
\hline
\end{tabular}

(D-OFDM) to stress that, in this solution, $\mathrm{CP}$ can be modified dynamically to adapt to the different TO.

The first two results (symbol error rate and throughput) are obtained from the hardware testbed, and assume a SISO case with perfect synchronization between transmitter and base station. Fig. 4 shows the symbol error rate with respect to the signal-to-interference-plus-noise (SINR) ratio. The purpose of presenting these result is identifying the SINR value where waveforms behave similarly in terms of symbol error rate; that SINR value will be used later as the working point when studying the impact of TO. The SINR in the received signal is obtained by estimating the power ratio between the part of the frequency spectrum of the received signal that contains only noise, and the part of the spectrum that contains both signal and noise; and averaging this value over a time interval. As shown in Fig. 4, D-OFDM behaves better than OQAM/FBMC and UF-OFDM for higher values of SINR (most likely, because both the non perfect reconstruction prototype filter implemented in OQAM/FBMC and the sampled Chebyshev filter, increase the SINR floor). Fig. 5 shows the throughput performance with respect to the SINR. As shown, while OQAM/FBMC and UF-OFDM achieve near $100 \%$ throughput for high values of SINR, in the OFDM case there is an upper bound because the longer the $\mathrm{CP}$ the lower the maximum throughput, a direct consequence of the reduced spectral efficiency.

Next, the hardware testbed is used to mimic the sidelink communication scenario of Fig. 1, where car \#1 and car \#3 are the transmitters and car \#2 is the receiver. Car \#1 and car \#3 transmit in the same carrier frequency but on two distinct nonoverlapping sub-bands (upper and lower, with 24 data carriers each). It is assumed that car \#2 perceives car \#1 transmission perfectly synchronized and car \#3 transmission with a certain TO. The TO is adjusted in $50 \mathrm{~ns}$ steps, which correspond to a degree of unsynchronization of 1 sample. Fig. 6 shows the BER performance with respect to this TO, at the SINR value where the symbol error rate of the waveforms in Fig. 4 is approximately similar (about $17.25 \mathrm{~dB}$ ). The target BER determines the highest tolerable TO. Note that, in case of 


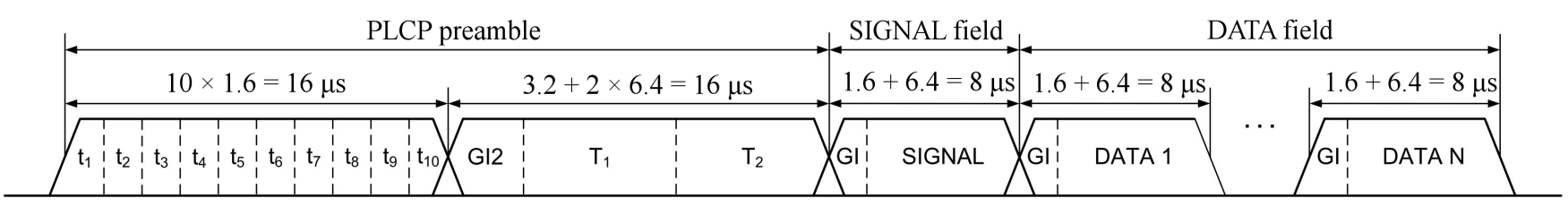

Fig. 3. IEEE $802.11 \mathrm{p}$ physical layer protocol data units (PPDU) frame.

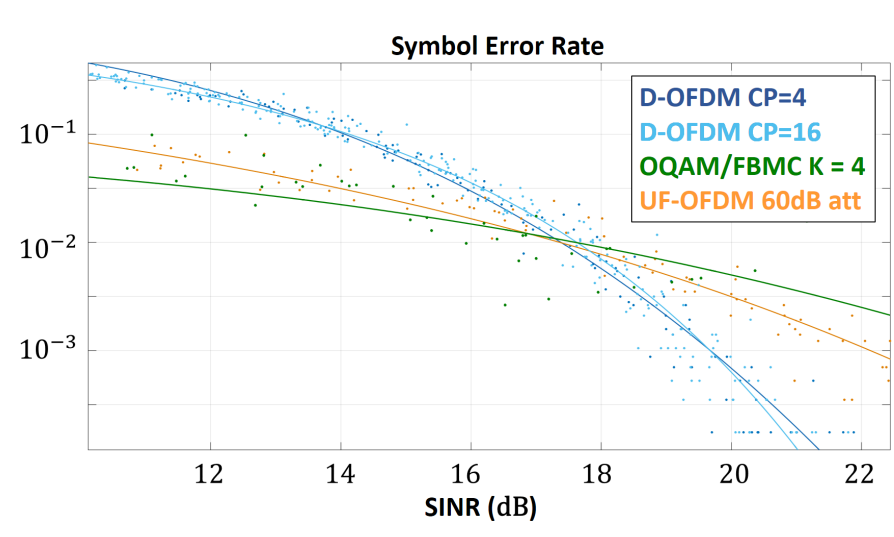

Fig. 4. Symbol error rate with respect to the signal-to-interference-plus-noise ratio.

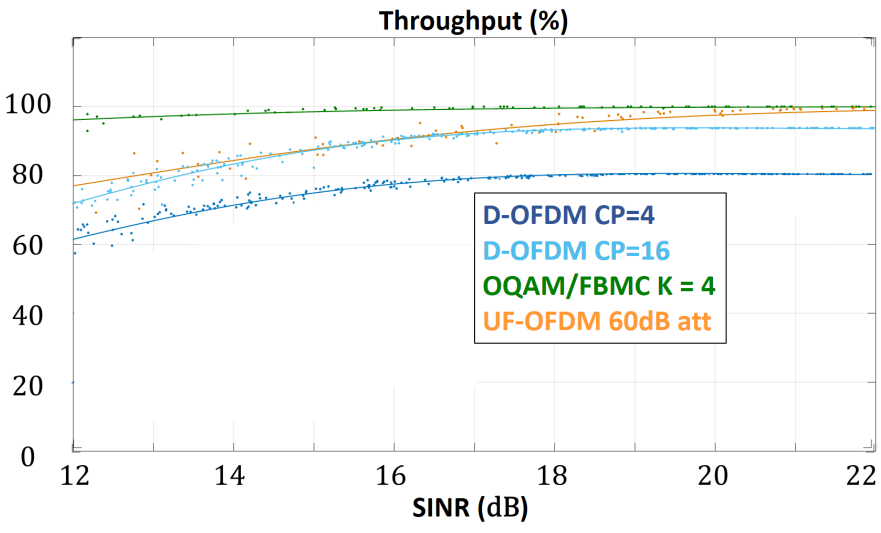

Fig. 5. Throughput performance with respect to the signal-to-interferenceplus-noise ratio.

OFDM, if car \#3 transmission is misaligned, then its TO is equal to the relative delay to car \#2. When the FFT operation is performed, car \#2 may experience ISI and ICI from car \#3 because, if the OFDM symbol of car \#3 does not lie within the FFT window of car \#2 the FFT duration may be extended over the symbol boundary, and if the OFDM symbol of car \#3 lies within the FFT window, then it will be now less robust to the delay spread of the channel. Note that the ISI and ICI from car \#3 cause performance degradation also to car \#1 transmission, which is perfectly synchronized. This multiple TO issue in OFDM may be solved by increasing the $\mathrm{CP}$ duration, so that it covers both the longest transmission delay and the channel's maximum delay spread; however this may require the use of TO estimation methods for OFDM. As

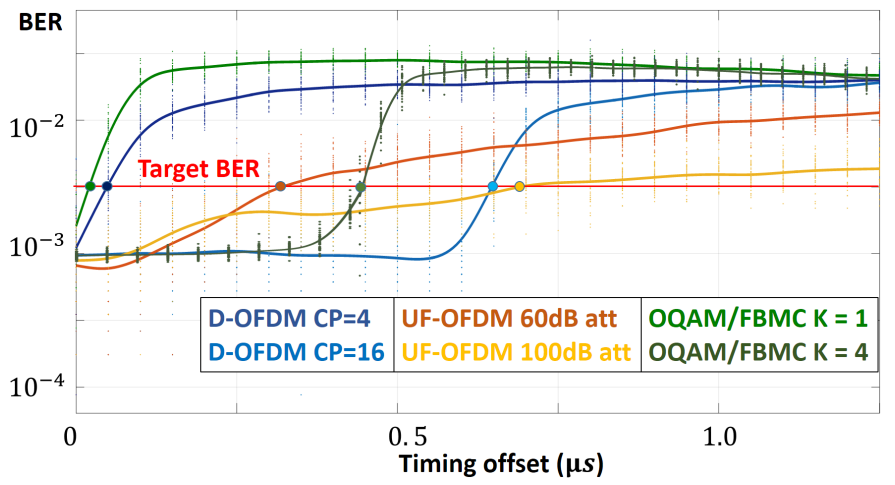

Fig. 6. BER performance with respect to the TO. The target BER determines the highest tolerable TO.

shown in Fig. 6, the receiver absorbs small positive TO (signal arrives later than the receiver expected) as long as the TO is within the duration of the $\mathrm{CP}$, approximately. However, OFDM performance degrades significantly as soon as the TO is such that the channel transient exceeds the $\mathrm{CP}$ duration. It is noteworthy that the experimental results of the effect of TO on OFDM are in concordance with the performance reported in [10] for positive TO because of the CP.

In Fig. 6, OQAM/FBMC exhibits a similar behavior and the signal is protected as far as the robustness provided by the overlap factor is not surpassed. In contrast, any TO in UF-OFDM systems affects the system performance, since no CP is inserted. Even with a small TO, the orthogonality between subcarriers is destroyed, which causes ICI, and ISI. However, for UF-OFDM the filter ramp-up/down may provide a soft protection against TO, since relatively small energy is contained, and as shown the degradation instead of sudden is progressive. In fact, increasing the robustness of UF-OFDM against TO is achieved by longer ramp-up/down periods. This protection is also applicable to OQAM/FBMC, but note that the results shown in Fig. 6 do not implement the rampup/down mechanism, and thus Fig. 6 must not be interpreted as stating that OQAM/FBMC is worse than UF-OFDM.

Finally, Fig. 7 shows the throughput performance comparison of SISO and MIMO 2x2 with respect to the SINR. In this case, the transmitter and receiver boards are located $1 \mathrm{~m}$ apart. The transmitter uses spatial multiplex (open loop/CSIR) MIMO with no precoding and, because of the distance, a sparse multipath channel is expected during the experiments. In order to achieve competitive MIMO results, OQAM/FBMC pilots are implemented according to [21]. The impact of the 


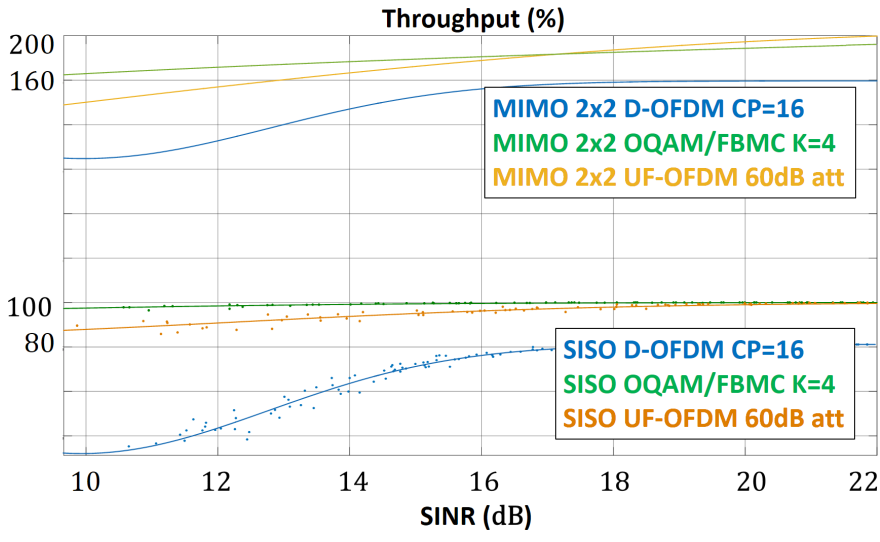

Fig. 7. Throughput performance comparison of SISO and MIMO $2 \times 2$ with respect to the signal-to-interference-plus-noise ratio in a MIMO.

$\mathrm{CP}$ on the throughput is also present and, as shown, for higher values of SINR the performance of UF-OFDM is slighthly better than that of OQAM/FBMC.

\section{CONCLUSIONS}

The hardware testbed presented in this paper is a valid framework for rapid physical layer prototyping, allowing arbitrary combinations of multiple-antenna transmitters and receivers. Although implementing an OFDM waveform is less complex, results show that OFDM is less robust against the lack of synchronization. However, there exist mechanisms that improve OFDM robustness (e.g. a longer $\mathrm{CP}$ or filtering, i.e. UF-OFDM). On the other hand, OQAM/FBMC is more robust against the lack of synchronization, but not as easily applicable to MIMO, since straightforward implementations of FBMC are more complex than OFDM. UF-OFDM is also robust against timing issues and has a complexity similar to OFDM. In any case, we have highlighted the need for a software defined radio approach to provide more flexibility to accommodate sidelink communication into a frame-structured cellular system.

Future work shall use the testbed to research the impact of mobility on the performance of FBMC, UF-OFDM (as well as other waveforms investigated by METIS-II) and check their degradation, including a performance assessment under different modifications to the numerology of the waveforms. Additionally, the $4 \times 4$ MIMO case shall be assessed, extending the SISO and $2 \times 2$ MIMO experiment results provided in this paper. Moreover, additional efforts should be made in order to harmonize the PHY layer with a single transmission chain that could implement a set of different waveforms.

\section{ACKNOWLEDGMENT}

Part of this work has been performed in the framework of the H2020 project METIS-II (H2020-ICT-2014-2, topic ICT-14-2014) co-funded by the European Commission. The authors would like to acknowledge the contributions of their colleagues. The views expressed are those of the authors and do not necessarily represent the project. Part of this work has been also supported by the Ministerio de Economía y Competitividad, Spain (TEC2014-60258-C2-1-R), by the European FEDER funds.

\section{REFERENCES}

[1] "Part 11: wireless LAN medium access control (MAC) and physical layer (PHY) specifications amendment 6: wireless access in vehicular environments". IEEE 802.11p published standard. IEEE. July 15, 2010. Retrieved August 10, 2011.

[2] D. Martín-Sacristán, J. F. Monserrat, J. Cabrejas-Peñuelas, D. Calabuig, S. Garrigas, N. Cardona, "On the way towards fourth-generation mobile: 3GPP LTE and LTE-advanced," EURASIP Journal on Wireless Communications and Networking, 2009.

[3] P. Siohan, C. Siclet, N. Lacaille, "Analysis and design of OFDM/OQAM systems based on filterbank theory," IEEE Transactions on Signal Processing, vol. 50, no. 5, pp. 1170-1183, August 2002.

[4] V. Vakilian, T. Wild, F. Schaich, S. Ten Brink, J.-F. Frigon, "Universalfiltered multi-carrier technique for wireless systems beyond LTE," in IEEE Globecom Workshops, 2013, pp. 223228.

[5] G. Jue, S. Shin, Keysight Technologies Implementing a flexible testbed for $5 G$ waveform generation and analysis, White Paper, Keysight Technologies, Apr. 20, 2015.

[6] M. Danneberg, R. Datta, G. Fettweis, "Experimental testbed for dynamic spectrum access and sensing of 5G GFDM waveforms," Vehicular Technology Conference, IEEE 80th, Vancouver, BC, 2014, pp. 1-5.

[7] F. Kaltenberger, R. Knopp, M. Danneberg, A. Festag, "Experimental analysis and simulative validation of dynamic spectrum access for coexistence of 4G and future 5G systems," Networks and Communications, European Conference on, Paris, 2015, pp. 497-501.

[8] K. W. Martin, "Small side-lobe filter design for multitone datacommunication applications," in IEEE Transactions on Circuits and Systems II: Analog and Digital Signal Processing, vol. 45, no. 8, pp. 1155-1161, Aug 1998.

[9] M. Bellanger, FBMC physical layer: a primer, PHYDYAS, Jan 2010.

[10] Y. Mostofi, D. C. Cox, "Mathematical analysis of the impact of timing synchronization errors on the performance of an OFDM system," in IEEE Transactions on Communications, vol. 54, no. 2, pp. 226-230, Feb. 2006.

[11] M.AA Hasan, F. Nabita, A, Khandakar, I. Ahmed, F. Ahmed, "Analytical evaluation of timing offset error in OFDM system," Communication Software and Networks, vol.3, no.7, pp.26-28, Feb. 2010.

[12] H. Saeedi-Sourck, Y. Wu, J. W. M. Bergmans, S. Sadri, B. FarhangBoroujeny, "Sensitivity analysis of offset QAM multicarrier systems to residual carrier frequency and timing offsets," Signal Process. 91 (July (7)) (2011) pp. 16041612

[13] H. Lin, M. Gharba, P. Siohan, "Impact of time and carrier frequency offsets on the FBMC/OQAM modulation", Signal Processing, vol. 102, pp. 151-162, 2014.

[14] T. Fusco, A. Petrella, M. Tanda, "Sensitivity of multi-user filter-bank multicarrier systems to synchronization errors," Communications, Control and Signal Processing, 3rd International Symposium on, St Julians, 2008, pp. 393-398.

[15] X. Wang, T. Wild, F. Schaich, "Filter pptimization for carrier-frequencyand timing-offset in universal filtered multi-carrier systems," Vehicular Technology Conference, 2015 IEEE 81st, Glasgow, 2015, pp. 1-6.

[16] Q. Bai, J. A. Nossek, "On the effects of carrier frequency offset on cyclic prefix based OFDM and filter bank based multicarrier systems," Signal Processing Advances in Wireless Communications (SPAWC), 2010 IEEE Eleventh International Workshop on, Marrakech, 2010, pp. 1-5.

[17] A. Aminjavaheri, A. Farhang, A. RezazadehReyhani, B. FarhangBoroujeny, "Impact of timing and frequency offsets on multicarrier waveform candidates for 5G”, arXiv:1505.00800v2 [cs.IT] 9 Sep 2015 [Online]. Available: http://arxiv.org/abs/1505.00800.

[18] Mango Communications, "WARP v3 Kit", [Online]. Available: https://mangocomm.com/products/kits/warp-v3-kit.

[19] T. M. Schmidl, D. C. Cox, "Robust frequency and timing synchronization for OFDM," IEEE Transactions on Communications, vol. 45, no. 12, pp. 16131621, Dec. 1997.

[20] C. Dick, F. Harris, "FPGA implementation of an OFDM PHY," in Proc. Conference Record of the Thirty-Seventh Asilomar Conference on Signals, Systems and Computers., vol. 1, Nov. 2003, pp. 905909.

[21] J.-P. Javaudin, D. Lacroix, A. Rouxel, "Pilot-aided channel estimation for OFDM/OQAM," in Vehicular Technology Conference, 2003. VTC 2003Spring. The 57th IEEE Semiannual, vol.3, no., pp.1581-1585 vol.3, 22-25 April 2003. 